\title{
Choriocarcinoma Metastatic to the Kidney Presenting With Prolonged Amenorrhea and Flank Pain
}

\author{
Fariba Behnamfar, ${ }^{1,}$ Fereshteh Mohammadizadeh, ${ }^{2}$ Leila Hashemi, ${ }^{3}$ and Somayeh Sheikhalian ${ }^{3}$ \\ ${ }^{1}$ Department of Obstetrics and Gynecology, School of Medicine, Isfahan University of Medical Sciences, Isfahan, IR Iran \\ ${ }^{2}$ Department of Pathology, School of Medicine, Isfahan University of Medical Sciences, Isfahan, IR Iran \\ ${ }^{3}$ School of Medicine, Isfahan University of Medical Sciences, Isfahan, IR Iran \\ "Corresponding author: Fariba Behnamfar, Department of Obstetrics and Gynecology, School of Medicine, Isfahan University of Medical Sciences, Isfahan, IR Iran. Tel: \\ +98-9133285642, E-mail: behnamfar_fa@med.mui.ac.ir
}

Received 2015 July 18; Accepted 2015 August 03.

\begin{abstract}
Introduction: Gestational choriocarcinoma usually occurs following an intrauterine pregnancy. We report a case of metastatic choriocarcinoma to the left kidney and lungs with long term intermittent amenorrhea and vaginal bleeding after a normal vaginal delivery.

Case presentation: A 43-year-old rural woman presented with prolonged amenorrhea. Her last delivery was three years ago. She also complained of hematuria and left flank pain. Serum $\beta$-hCG level was considerably high. She underwent endometrial curettage. Pathologic examination of endometrial curettage specimen revealed choriocarcinoma. Ultrasound revealed enlarged uterus involved by an irregular mass with heterogenous echo pattern and extensive myometrial invasion. A mass with similar echo pattern was also evident in the left kidney. Computerized tomography confirmed the intrauterine mass and involvement of the left kidney. On chest X-ray, metastatic nodules were seen in both lungs and in the left retrocardiac space. The patient underwent 10 courses of chemotherapy (8 treatment courses and 2 courses for consolidation) with EMA-CO regimen (etoposide, methotrexate, actinomycin D, cyclophosphamide, and vincristine/oncovine). Following the fifth course of chemotherapy, she became pancytopenic and febrile. This condition was successfully managed with G-CSF, leukovorin and antibiotics. The patient is now well and still under the chemotherapy. Her serum $\beta$-hCG level has fallen to negative (3 IU/mL).

Conclusions: Gestational trophoblastic diseases should be considered in the differential diagnosis of prolonged amenorrhea in patients of reproductive age with a history of prior pregnancy. Moreover, symptoms related to metastatic involvement such as hematuria and flank pain may be among the first clinical manifestations of choriocarcinoma.
\end{abstract}

Keywords: Choriocarcinoma, Amenorrhea, Metastasis

\section{Introduction}

Gestational choriocarcinoma is most commonly seen in women of reproductive age, generally within the first year after pregnancy. It usually occurs following an intrauterine pregnancy. Although this condition most frequently originates from a molar pregnancy, it may also occur following an abortion or a normal delivery. Patients with intrauterine choriocarcinoma frequently present with abnormal uterine bleeding (1). Less commonly, they may present with amenorrhea and even unusual clinical presentations such as acute abdomen resulting from spontaneous uterine perforation, hematuria, hemothorax, or fetomaternal hemorrhage (1-5). About 30 percent of the patients with choriocarcinoma initially present with metastatic disease and lung is the most common site of metastatic involvement $(1,6)$. Renal metastasis may result in oliguria, hematuria or massive retroperitoneal hemorrhage (7). Renal metastases are almost invariably pre- ceded by pulmonary metastases. This seems to be the result of dissemination of tumor cells from lung metastasis through the general circulation (7).

Herein, we report a case of choriocarcinoma presenting with prolonged amenorrhea followed by hematuria and left flank pain after a normal vaginal delivery.

\section{Case Presentation:}

A 43-year-old rural woman (G7L6EP1) presented with intermittent amenorrhea and vaginal bleeding since her last normal vaginal delivery three years ago. Later, the patient complained of a persistent vaginal bleeding for about two months as well as hematuria and left flank pain. The patient underwent endometrial curettage. On microscopic examination of the curettage material, sheets of cytotrophoblasts and syncytiotrophoblasts were seen growing in a plexiform pattern in the background of hemorrhage and necrosis. The trophoblastic cells showed nuclear atypia 
and mitotic activity (Figure 1). No chorionic villi were evident. The patient was then referred to our center for treatment of choriocarcinoma. At the time of admission in our hospital, the patient had a large uterus and left flank tenderness on physical examination. Serum $\beta$-hCG level was $1500 \mu \mathrm{IU} / \mathrm{mL}$ at the time of admission and rised to 10000 $\mu \mathrm{IU} / \mathrm{mL}$ before beginning the treatment. Abdominal and pelvic sonography revealed a large uterine mass with myometrial invasion and heterogenous echo pattern measuring $57 \times 36 \mathrm{~mm}$ in the fundus and body. A $126 \times 82 \mathrm{~mm}$ mass lesion was also seen in the middle pole of the left kidney. Computerized tomography of the abdominopelvis showed a large hemorrhagic mass in the left kidney confined to the fascia gerota (Figure 2). Two enlarged lymph nodes were also evident in the left renal hilus. Metastases to the liver and spleen were absent. On chest X-ray, multiple nodules were seen in both lungs and in the left retrocardiac space (Figure 3). Upper and lower gastrointestinal endoscopy were normal. Brain imaging revealed no brain metastasis. According to world health organization prognostic index for gestational trophoblastic disease, our patient fitted into the high risk category with a total score of 13 (age score: 1 , antecedent pregnancy: 2 , interval: $4, \beta$-hCG: 1, largest tumor: 2 , site of metastases: 1 , number of metastases identified: 2) (8). Chemotherapy with standard EMACO regimen (etoposide, methotrexate, actinomycin D, cyclophosphamide, and vincristine/oncovine) was planned for the patient. Her general status was checked weekly by complete blood count, serum BUN and creatinine and liver function tests. Serum $\beta$-hCG level was also measured weekly. After receiving five courses of chemotherapy, the patient became febrile and pancytopenic. This condition was successfully managed with G-CSF, leukovorin and antibiotics. After 8 courses of chemotherapy, her serum $\beta$ hCG level fell to $3 \mu \mathrm{IU} / \mathrm{mL}$. She is now in remission and is followed with serial serum $\beta$-hCG measurements.

\section{Discussion}

The case we reported presented with prolonged amenorrhea followed by left-sided flank pain and gross hematuria. Although 50 percent of gestational choriocarcinomas are preceded by a molar pregnancy, our patient lacked such a history and developed choriocarcinoma following a normal vaginal delivery.

Patients with high risk metastatic gestational trophoblastic neoplasia should be first treated by multiagent chemotherapy. Standard EMA/CO regimen is highly effective for treatment of high-risk gestational trophoblastic neoplasia (6). Wang et al. (7) have reviewed 448 cases of choriocarcinoma admitted in their center in a 30 year period. The incidence of renal metastasis was $6.9 \%$ in the

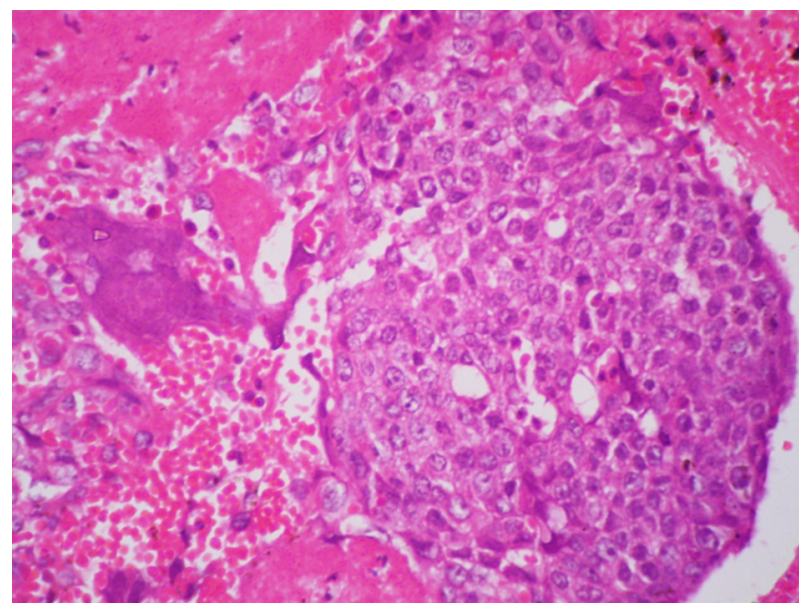

Figure 1. Sheets of Cytotrophoblasts and Syncytiotrophoblasts Growing in a Plexiform Pattern in the Background of Hemorrhage (H \& E Staining; $\times 10$ Objective)

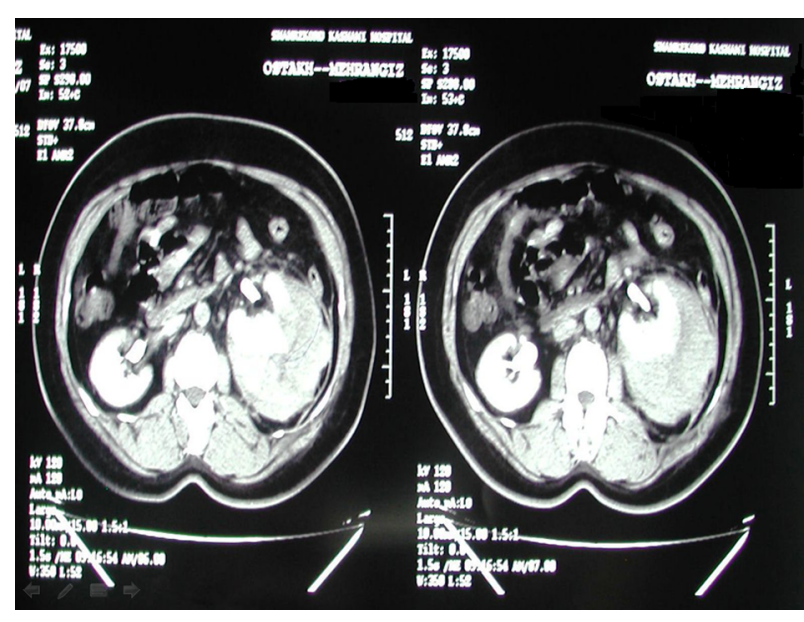

Figure 2. Abdominopelvic CT Scan Showing Left Kidney Mass

studied population. These renal metastases were found to be very sensitive to chemotherapy. This may be attributable to the high drug concentration in the renal tissue during their excretion (7).

Adjuvant surgical procedures may be indicated in chemotherapy-resistant high-risk gestational trophoblastic neoplasia. These procedures especially include hysterectomy and pulmonary resection of chemotherapy resistant metastatic foci of choriocarcinoma in the lung. Surgical intervention may also become necessary to control hemorrhage (8). In our case, complete remission of disease was achieved with chemotherapy alone and without need to do any of these surgical procedures.

In conclusion, gestational trophoblastic diseases 


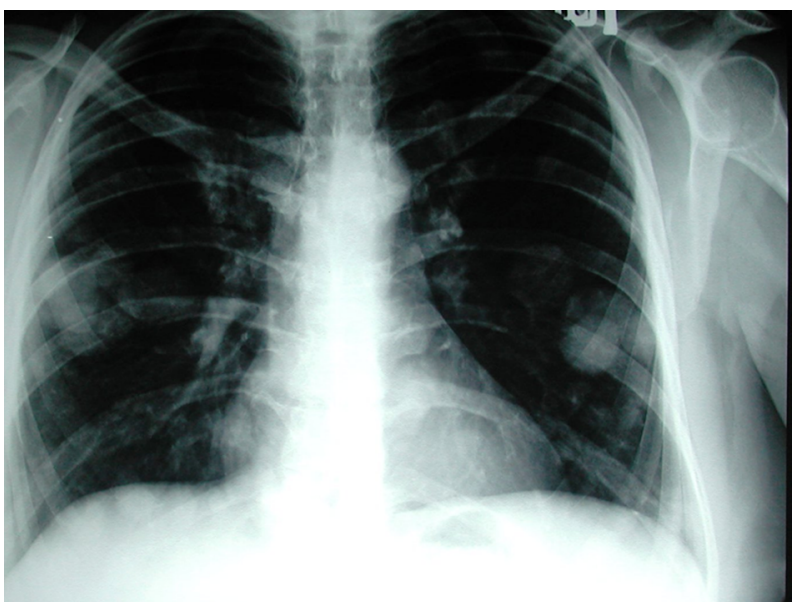

Figure 3. Chest X-Ray Showing Bilateral Nodules in the Lungs

should be considered in the differential diagnosis of prolonged amenorrhea in patients of reproductive age with a history of prior pregnancy. Moreover, symptoms related to metastatic involvement such as hematuria and flank pain may be among the first clinical manifestations of choriocarcinoma. Metastatic lesions found in any organ including kidneys in parous women of reproductive age should raise the possibility of choriocarcinoma Standard EMA/CO regimen is usually the choice treatment in pa- tients with high-risk gestational trophoblastic neoplasia.

\section{References}

1. Saha K, Basuthakur S, Jash D, Bandyopadhyay A. Gestational choriocarcinoma presenting as hemothorax. Indian J Med Sci. 2010;64(5):237-40. doi: 10.4103/0019-5359.98962. [PubMed: 22842324].

2. Kairi-Vassilatou E, Papakonstantinou K, Grapsa D, Kondi-Paphiti A, Hasiakos D. Primary gestational choriocarcinoma of the uterine cervix. Report of a case and review of the literature. Int J Gynecol Cancer. 2007;17(4):921-5. doi: 10.1111/j.1525-1438.2006.00852.x. [PubMed: 17309671].

3. Liberis V, Bouchlariotou S, Ammari A, Psillaki A, Ntatidou M, Sivridis E, et al. Acute abdomen as initial presentation of gestational choriocarcinoma. Arch Gynecol Obstet. 2009;280(5):859-62. doi: 10.1007/s00404009-0968-5. [PubMed: 19288267].

4. Zajaczkowski T, Straube W, Kissler W, Schumacher T, de Dycker RP. [Spontaneous kidney rupture]. Urologe A. 1990;29(5):256-60. [PubMed: 2219600].

5. Touboul C, Faivre E, Boithias C, Mass AE, Senat MV, Fernandez H, et al. [Fetomaternal hemorrhage caused by intraplacental choriocarcinoma]. J Gynecol Obstet Biol Reprod (Paris). 2010;39(2):156-8. doi: 10.1016/j.jgyn.2009.10.008. [PubMed: 20074866].

6. Turan T, Karacay O, Tulunay G, Boran N, Koc S, Bozok S, et al. Results with EMA/CO (etoposide, methotrexate, actinomycin D, cyclophosphamide, vincristine) chemotherapy in gestational trophoblastic neoplasia. Int $J$ Gynecol Cancer. 2006;16(3):1432-8. doi: 10.1111/j.1525-1438.2006.00606.x. [PubMed: 16803542].

7. Wang YE, Song HZ, Yang XY, Dong SY, Gan N. Renal metastases of choriocarcinoma. A clinicopathological study of 31 cases. Chin Med J (Engl). 1991;104(9):716-20. [PubMed: 1935350].

8. Lurain JR, Singh DK, Schink JC. Role of surgery in the management of high-risk gestational trophoblastic neoplasia. J Reprod Med. 2006;51(10):773-6. [PubMed: 17086805]. 